\title{
SISTEMA DE MONITORAMENTO DE TEMPERATURA, UMIDADE E LUMINOSIDADE PARA USO EM PEQUENAS PLANTAÇÕES DO SERTÃO PARAIBANO.
}

\author{
DOI: 10.37702/2175-957X.COBENGE.2021.3652
}

Natanael Cruz de Souza - natanael.ytr@gmail.com

Instituto Federal da Paraiba IFPB

Patos sn

58709-000 - Patos - PB

JOSE DIEGO FERREIRA DA SILVA - diego19913011@gmail.com

Instituto Federal da Paraiba IFPB

Rua Luiz Linderman de Queiroz 147

58706-044 - Patos - PB

Thamyris da Silva Evangelista - thamyris.tse@gmail.com

Instituto Federal da Paraíba

Rua Hélio Rodrigues Ferreira 200

58077-125 - João Pessoa - PB

Michelle Ferreira Leite - michelle.leite@ifpb.edu.br

Instituto Federal da Paraíba

Rua João José da Costa 131

58075-030 - João Pessoa - PB

Daniel dias dos Santos - danieldias.ufpb@gmail.com

Universidade Federal da Paraíba

Rua Hélio Rodrigues Ferreira 200

58077-125 - João Pessoa - PB

Resumo: A constante evolução tecnológica possibilita o uso da eletrônica para melhorar as condições de trabalho, a fim de evitar desperdícios, prejuízos e aumentar a produtividade. Na área da agricultura, a eletrônica pode ser usada para obter as informações necessárias que uma planta necessita para se desenvolver, como temperatura, umidade do solo, luminosidade, entre outros. Neste cenário, o foco principal desse estudo foi desenvolver um sistema de 
monitoramento utilizando sensores e Arduino que possibilitasse o usuário de baixa renda a ter a possibilidade de melhorar seu meio de cultivo. Utilizando a plataforma Tinkercad foi possível realizar as simulações que que monitoram os fenômenos físicos e futuramente se tornariam um protótipo chamado de Monitor T.U.L, este controle é feito utilizando o Arduino UNO e alguns sensores. Com isso foi possível alcançar o objetivo de um protótipo que se mostrou bastante eficaz na coleta de dados e na automação utilizando relés, de baixo custo e de fácil integração com o Arduino. Para continuação busca-se o aprimoramento do mesmo, com melhora no código, na alimentação e com a criação de um aplicativo que atuará junto ao monitor T.U.L facilitando ainda mais a coleta e transmissão de dados

Palavras-chave: Arduino. Sensores. Monitoramento. Sertão da Paraíba. 


\section{SISTEMA DE MONITORAMENTO DE TEMPERATURA, UMIDADE E LUMINOSIDADE PARA USO EM PEQUENAS PLANTAÇÕES NO SERTÃO PARAIBANO}

\section{INTRODUÇÃO}

Uma grande dificuldade presente na educação é aliar o conhecimento adquirido em sala de aula com a prática. Os alunos, muitas vezes, não conseguem compreender o conteúdo de uma maneira efetiva, sem as atividades experimentais atreladas ao processo de ensino. Assim, executar atividades práticas é de fundamental importância para o processo de ensino-aprendizagem.

Aliar as atividades práticas à aprendizagem baseada em problemas - ABP torna-se ainda mais interessante para o processo de formação dos estudantes. A técnica denominada aprendizagem baseada em problemas é um modelo de aprendizagem dita como um recurso didático centrado no aluno, fazendo deste o principal agente responsável pelo seu aprendizado. Nessa metodologia, os alunos são expostos a projetos desafiadores com o objetivo de estimular a busca de soluções para os problemas apresentados e, assim, alcançar, de uma forma atrativa e inovadora, conhecimentos e habilidades que thes serão úteis na sua vida acadêmica e profissional (SOUZA e DOURADO, 2015; SILVA, L. et al, 2018; DIAS, Y et al, 2018).

Com base nisso, o objetivo deste artigo é apresentar um protótipo de monitoramento utilizando sensores e Arduino, que facilita as tarefas nas pequenas plantações, com o controle de umidade, temperatura e luminosidade, por meio do uso da plataforma de prototipagem eletrônica Arduino, como um estudo de caso para um problema desenvolvido baseada no ABP, utilizando materiais de baixo custo e sucatas. O protótipo foi idealizado por alunos do curso técnico em eletrotécnica do Instituto Federal da Paraíba - Campus Patos.

A cidade de Patos é um município brasileiro no estado da Paraíba, localizado na mesorregião do Sertão Paraibano. O clima de Patos é semiárido quente e seco, com poucas chuvas. A temperatura média compensada anual é de $27,6{ }^{\circ} \mathrm{C}$ e 0 índice pluviométrico de 764 milímetros $(\mathrm{mm})$, com chuvas concentradas entre janeiro e abril, Patos se encontra na 18 ${ }^{\text {a }}$ colocação no ranking das 20 cidades mais quentes do Brasil (INMET, 2019). A vegetação predominante na cidade é a Caatinga, bioma ambiental, exclusivamente brasileiro, perfeitamente adaptado ao clima quente e seco, bastante sofrido pela degradação e intempéries (IBGE, 2019).

Com um clima seco e poucas chuvas, usar a tecnologia para que os agricultores possam implantar recursos de forma mais eficiente e monitorar toda sua plantação tornase essencial. O monitoramento das plantações, que podem incluir diversos fatores como, temperatura, umidade relativa do ar, umidade do solo, entre outros, é muito importante para que se possam tomar ações preventivas a fim de evitar perdas nas plantações, bem como aumentar a qualidade do alimento. Muitas vezes, o agricultor não consegue monitorar alguns destes fatores climáticos, para saber em que momento e em que quantidades utilizar agrotóxicos que combatam algumas pragas e doenças, ou mesmo realizar as irrigações (SHASHWATHI; BORKOTOKY; SUHAS, 2012). Além disso, muitos dispositivos existentes no mercado para realização dessas tarefas são de custo elevado, assim diversos pesquisadores têm sido motivados a desenvolver um sistema de automação baseado em Arduino. 
A relevância desta pesquisa, além da sua importância tecnológica e social está vinculada a aliar os conhecimentos adquiridos em sala de aula com a prática, pois uma das grandes dificuldades na educação é fazer a ponte entre o conhecimento ensinado em sala de aula com a prática, gerando, muitas vezes, a apatia do aluno com o assunto abordado.

Os conceitos que, se vistos somente textualmente, não são plenamente compreendidos pelos discentes, surgindo, fatalmente, lacunas de conhecimento na formação educacional dos mesmos. Desse modo, a realização de atividades experimentais, aliada à aprendizagem baseada em problemas como as propostas neste trabalho é extremamente importante no processo de ensino-aprendizagem, contribuindo, significativamente, para o crescimento do ensino e, consequentemente, da ciência (EVANGELISTA, 2016).

Este artigo está dividido em cinco seções, o qual na segunda seção são apresentados os conceitos sobre Arduino e sobre os sensores utilizados. Na terceira seção são apresentados a simulação e a montagem do protótipo. Na quarta seção, os resultados experimentais são apresentados e discutidos, e por fim, na quinta seção, as considerações finais deste trabalho

\section{REFERENCIAL TEÓRICO}

\subsection{Microcontrolador Arduino}

No Arduino, Figura 1, o processamento ocorre por meio de um microcontrolador Atmel AVR, sendo programado em linguagem similar ao $\mathrm{C} / \mathrm{C}++$. Possui uma interface de programação, denominada ambiente de desenvolvimento integrado (IDE), e, permite a elaboração de programas com inúmeros exemplos, e adquiridos de forma gratuita diretamente na página do desenvolvedor (OLIVEIRA; CLÁUDIO; ZANITTI; HUMBERTO, 2015).

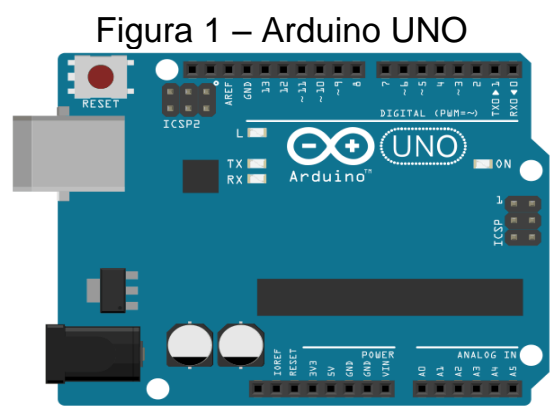

Fonte: embarcados.com.br/

\subsection{Sensores}

O Sensor é um elemento, dispositivo ou sistema que converte um mensurando físico, químico ou biológico de interesse em sinal óptico ou elétrico, de forma que possa ser transmitido ou processado (WERNECK, 1996). Neste trabalho estão sendo utilizados sensores de: temperatura, umidade e luminosidade.

Para o uso de sensores com a finalidade de monitorar automaticamente a temperatura, os sensores mais adequados são os dotados de saídas elétricas (tensão ou corrente) podendo ter transmissão de sinais na forma analógica ou digital (STEIDLE NETO, 2005). Um exemplo de sensor de temperatura de alta precisão é o LM 35, Figura 2, fabricado em circuito integrado pela National Semiconductor com três terminais, um de alimentação, um para a tensão de saída e um terminal neutro. A tensão de saída produzida é linear e proporcional a escala Celsius, sendo a resolução ajustada a tensão de saída, assim, por padrão do sensor LM 35 , a cada $10 \mathrm{mV}$ de tensão de saída a temperatura será de $1^{\circ} \mathrm{C}$, e assim por diante (LM35, 2019). 
Figura 2 - Sensor LM35.

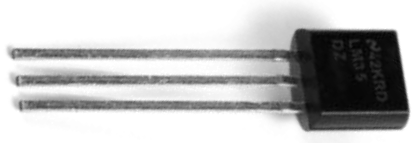

Fonte: eletrogate.com

Existem diversos métodos para medição de umidade do solo, cada um com suas particularidades e aplicações. Alguns parâmetros como precisão, tempo de leitura e custo para medição devem ser considerados na escolha do método que vai ser utilizado (SOUZA Mendes, 2006). Um sensor bastante utilizado é o HW-103, composto separadamente por uma sonda de detecção de umidade do solo e seu módulo para comunicação com o microcontrolador por meio de saída digital, que pode ser controlada utilizando o trimpot, um potenciômetro em miniatura ajustável, e emite um valor fixo de $0 \quad \mathrm{~V}$ ou $5 \mathrm{~V}$, e uma saída analógica, que emite uma tensão variável de 0V a 5V, Figura 3.

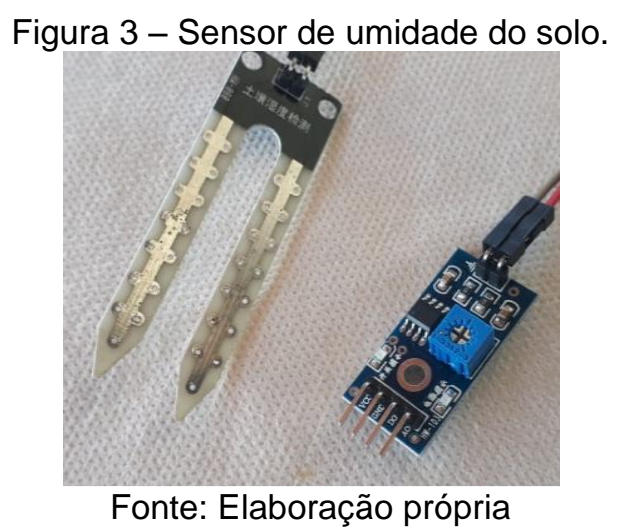

Com relação a luminosidade, o sensor utilizado neste trabalho foi o fotoelétrico, que tem por finalidade converter um sinal luminoso em um sinal elétrico. A Figura 4 apresenta um sensor LDR também conhecido como fotorresistência, pois varia sua resistência de acordo com a intensidade luminosa incidente nele, sendo assim, quanto maior o diâmetro do sensor maior será sua capacidade de controlar correntes mais intensas que passam por ele (WENDLING, 2010).

Figura 4 - Sensor LDR.

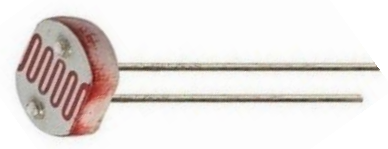

Fonte: eletrogate.com

\section{PROTÓTIPO DESENVOLVIDO}

Inicialmente, as simulações foram realizadas utilizando o site Tinkercad, ferramenta online e gratuita de simulação de circuitos elétricos e de modelagem em 3D, conforme apresentado na Figura 5. 
Figura 5 - Layout utilizando os componentes na simulação.

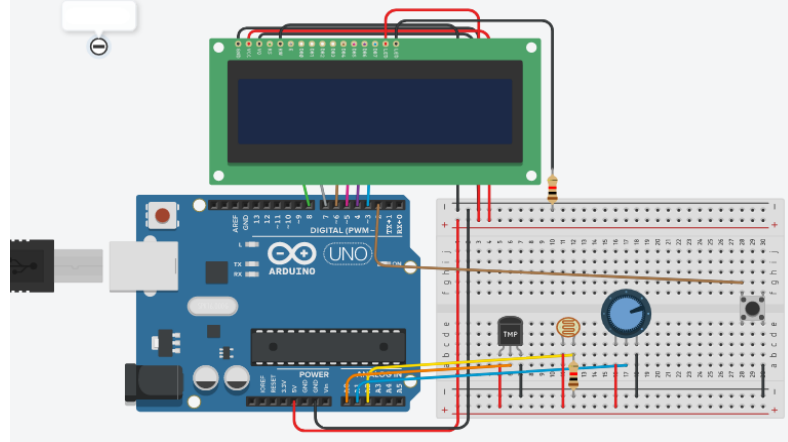

Fonte: tinkercad.com

Após a montagem foi realizada a criação do código para coletar informações dos sensores, converter essas informações e apresentá-las no display.

$\mathrm{Na}$ figura 6 é apresentado o layout do projeto comentado.

Figura 6 - Layout comentado.

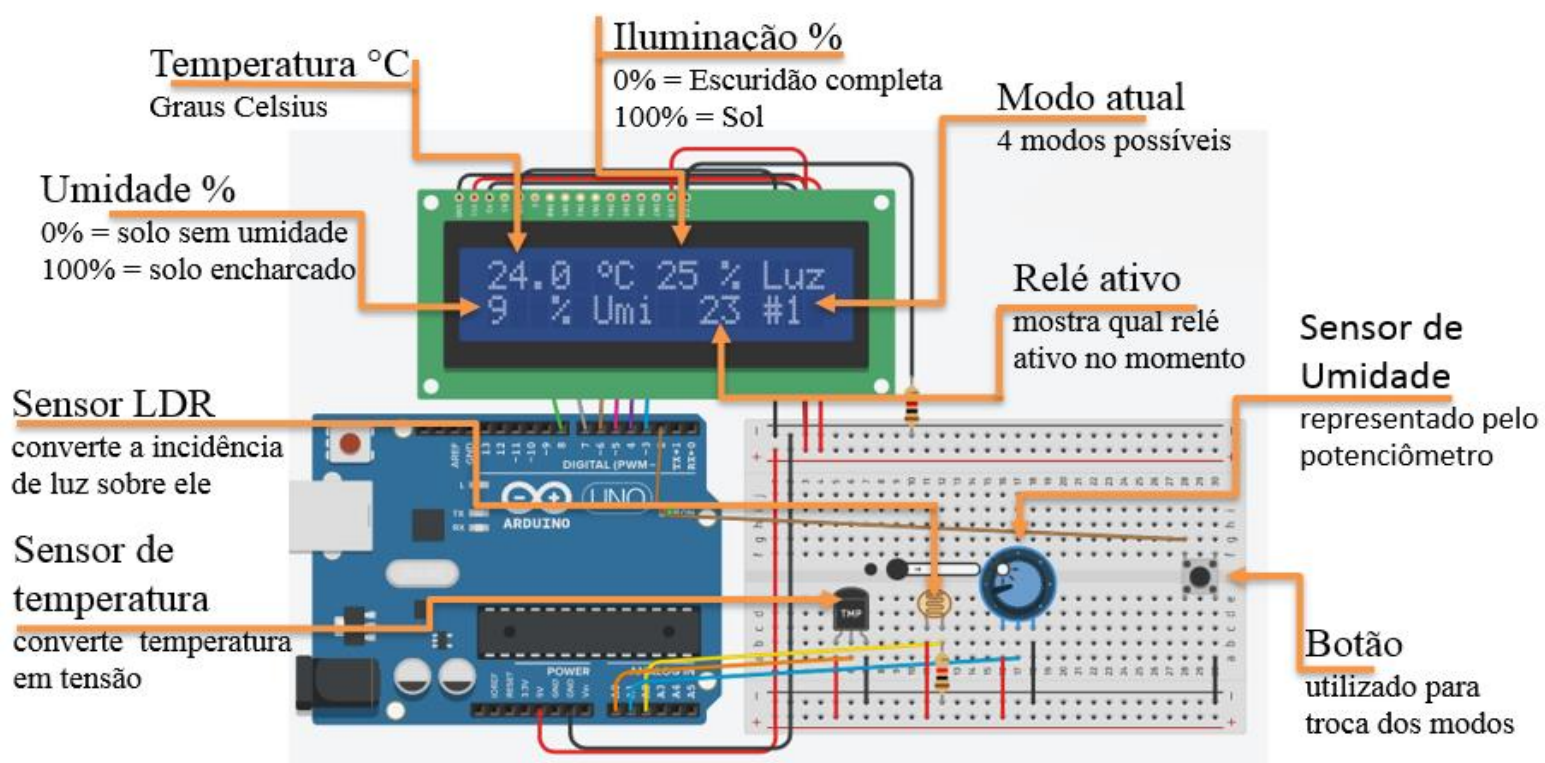

Fonte: Desenvolvida pelos autores.

Após as simulações, foi executada a parte experimental com a montagem física do circuito, conforme apresentado na Figura 7.

Figura 7 - Montagem e teste inicial.

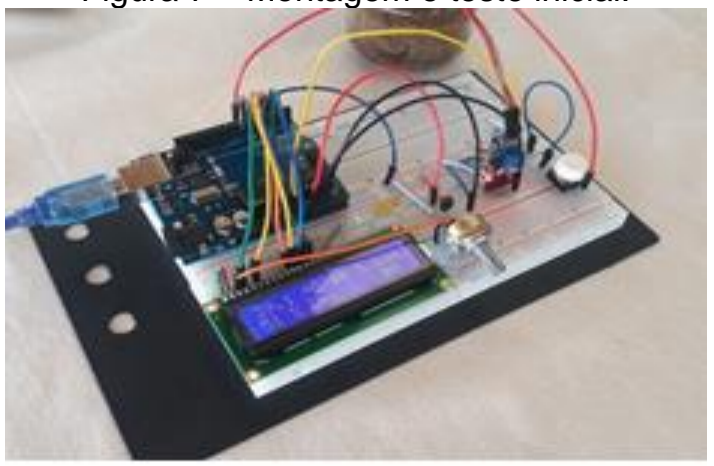

Fonte: Elaboração própria. 


\section{RESULTADOS E DISCUSSÃO}

Após o desenvolvimento inicial do projeto, começaram os testes práticos para verificar a estabilidade e precisão dos sensores. Inicialmente colocamos o protótipo em um ambiente controlado, o ambiente em questão era o laboratório de eletrotécnica do IFPB Campus Patos no qual era possível ter o controle da temperatura por meio do arcondicionado e da iluminação por meio do sistema de iluminação. Utilizamos um recipiente com terra e uma planta, a qual foi usada para testar o sensor de umidade, como apresentado na Figura 8.

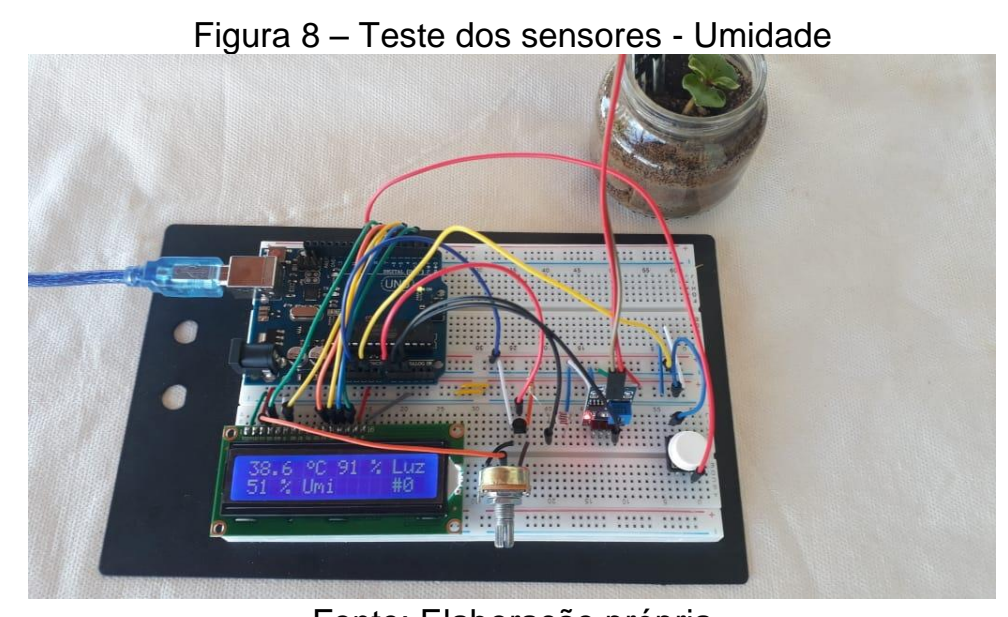

Fonte: Elaboração própria

Ao colocarmos o sensor de umidade no solo foi visto que ele apresentou $51 \%$ de umidade, Figura 8. Ao acrescentar um certo nível de água na terra essa umidade aumentou de valor indo para $70 \%$ até estabilizar. Foi verificado que o sensor de umidade estava seguindo corretamente a programação.

Na figura 9 marcado pelas setas laranjas, testamos o sensor de luminosidade, que se mostrou bastante eficaz na captação de luz, de maneira que é possível obter valores $0 \%$ para a escuridão completa e $100 \%$ para a claridade em um dia de sol.

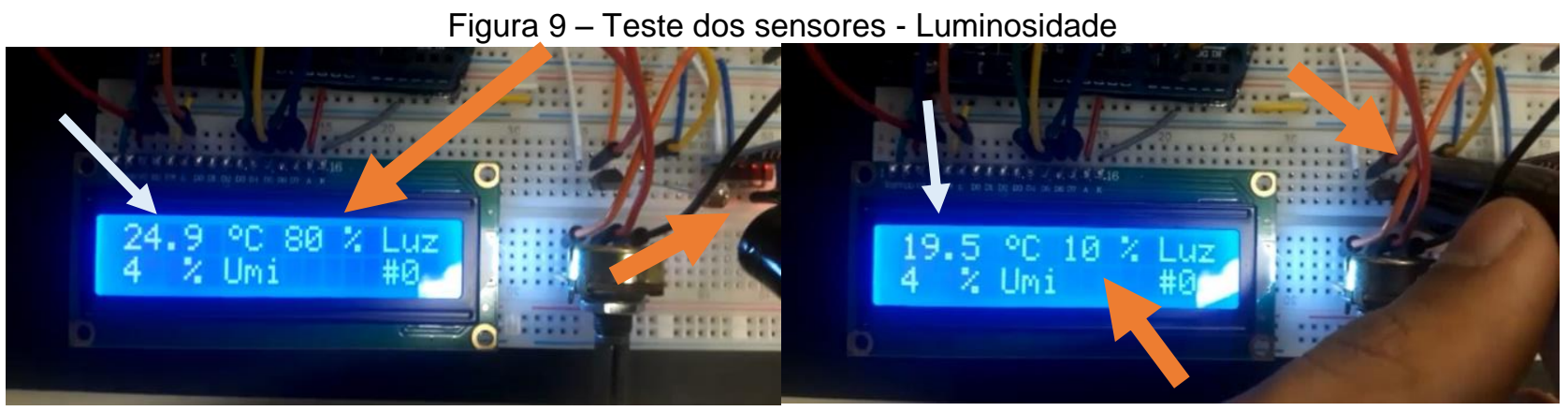

Fonte: Elaboração própria.

Para calibração e comparação do nosso protótipo, utilizamos um mini termômetro digital, visto na figura 10, ele é facilmente encontrado em lojas digitais por preços baixos e a sua precisão é de $\pm 1^{\circ} \mathrm{C}$. Com relação ao sensor de temperatura tivemos problemas em relação a sua precisão, como é visto na Figura 9 marcado pela seta branca, pois como utilizamos uma protoboard ela acarretou alguns erros de leitura. Estes erros são provenientes do mal contato entre os terminais dos sensores com a protoboard e até 
mesmo devido a estática presente no ambiente. Para o teste, colocamos o protótipo em dois tipos de ambiente, o primeiro foi o próprio laboratório de eletrotécnica no qual era um ambiente mais frio e o segundo local foi fora do laboratório, em um corredor, o ambiente mais quente, e então comparamos os valores do protótipo com o mini termômetro.

Figura 10 - Tl8009 Icd termômetro digital.

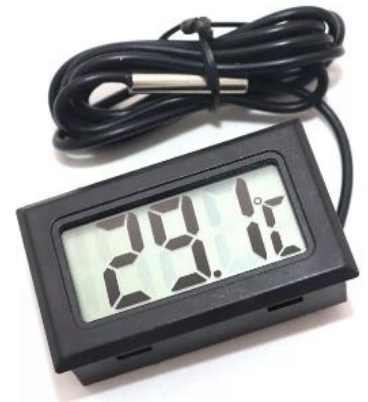

Fonte: Aliexpress.com

\subsection{Novas funções e componentes.}

Em seguida, novas funcionalidades e componentes foram adicionados ao trabalho: Adicionamos quatro padrões de modos de cultivo ao código, cada um deles tenta automatizar o processo de cultivo da plantação, estes padrões são citados e esclarecidos mais a frente. Também foi adicionado um componente eletromecânico chamado "Relé", Figura 11, o seu papel é o de um interruptor eletromecânico e seu funcionamento baseasse na seguinte ideia: Ao receber um sinal elétrico positivo no seu pino de dados, será acionada a sua bobina interna mudando assim a conexão dos terminais de carga. O relé possui três terminais para serem usados na carga, sendo o 4 normalmente fechado, 2 comum e o 3 normalmente aberto, Figura 11. Então, fizemos a seguinte conexão: terminal 5 ligado a uma porta digital do Arduino, terminal 1 aterrado ao GND do Arduino, desta maneira o Arduino irá controlar o relé. Para a carga temos: terminal 2 conectado ao positivo da fonte de alimentação, terminal 3 conectado ao terminal positivo da nossa carga (em que irá alimentar a carga) e o terminal 4 não será utilizado.

- Obs.: em alguns modelos de módulos relé, deve ser empregada uma alimentação (que normalmente é $5 \mathrm{v}$ ) para o terceiro terminal de entrada do modulo, isso é facilmente observado no próprio modulo ou no datasheet do mesmo.

Figura 11 -Módulo Relé.

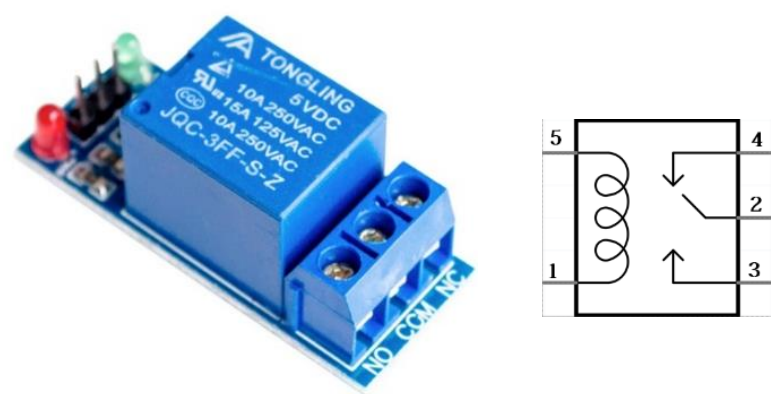

Fonte: multilogica-shop.com

A ideia de implementar os "modos de cultivo" partiu do princípio de que existem pequenos agricultores que produzem variados tipos de plantações, sejam elas de: milho, feijão, morangos, batatas, hortaliças (como coentro, alface, pimenta, cebolinha) entre outros. Estes tipos de plantas necessitam de diferentes cuidados para um crescimento 
saudável, então os 4 modos de cultivo tentam trazer ao agricultor a praticidade e agilidade de alternar entre estes tipos de plantações com mais facilidade e comodidade utilizando o mesmo equipamento de monitoramento.

Descrição dos modos de cultivo inicialmente desenvolvidos:

- Modo 1 é útil para irrigação ou resfriamento com precisão e economia, pois não irá gerar gastos além do necessário, seja de água ou energia. Neste como, os relés são sensíveis aos sensores, ou seja, se a temperatura em determinado momento for maior que a definida no programa, o relé número 1 irá ser acionado imediatamente acionando também o objeto conectado ao relé, caso contrário ele será desativado imediatamente.

- O modo 2 é semelhante ao modo 1: Pode ser utilizado para uma irrigação ou resfriamento com mais cuidado para com o objeto conectado ao relé (ex.: Bomba de água), pois assim ele trabalha em ciclos curtos. Este modo também é útil para uma melhor absorção de liquido por parte do solo. Entretanto, esse modo possui um delay que é usado para criar pausas durante o processo. Por exemplo, se o sensor de temperatura detectou que o ambiente está com a temperatura acima do programado, então será acionado o relé número 1 e este ficará acionado por alguns minutos, após esse tempo, ele será desativado pelo mesmo tempo, então o ciclo se repete até a mudança da temperatura.

- No modo 3 todos os relés são ativados independente dos valores dos sensores, esse modo é utilizado para teste dos relés e dos objetos conectados a eles.

- O modo 4 é utilizado para ativar os sensores via aplicativo.

Os modos de cultivo foram adicionados via código. Todos os modos atendem um propósito específico (como na descrição deles) e após os testes e ajustes eles funcionaram perfeitamente, com exceção do modo 4 que seria necessário o acoplamento do módulo bluetooth.

Caso o usuário necessite mudar a configuração atual basta pressionar o botão apresentado na Figura 12, que o Arduino irá alternar entre os modos de cultivo. Isto gera facilidades para o usuário dinâmico, aquele que está em constante mudança e possui diferentes tipos de plantações que necessitam de cuidados diferentes.

Figura 12 - Troca de modos de cultivo.

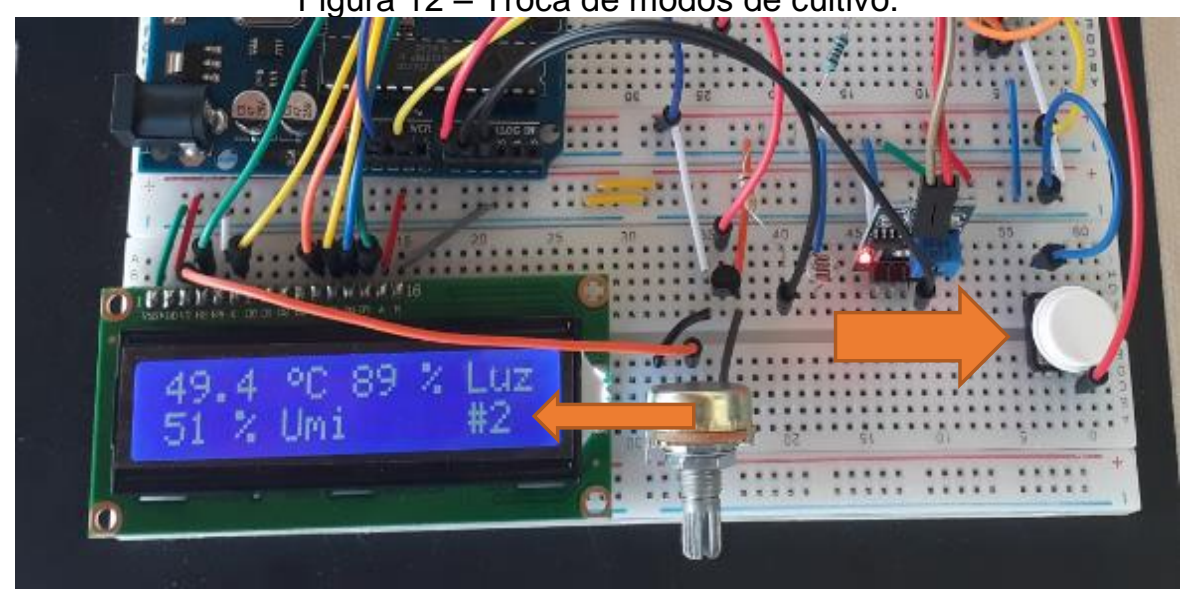

Fonte: Elaboração própria. 
Em seguida, é apresentado a construção de uma estufa para simular o ambiente real de plantação. Utilizamos materiais reciclados tais como: garrafa pet, papelão, palitos de churrasco, potes de plástico, conforme apresentado na Figura 13.

Figura 13 - Criação da estufa.

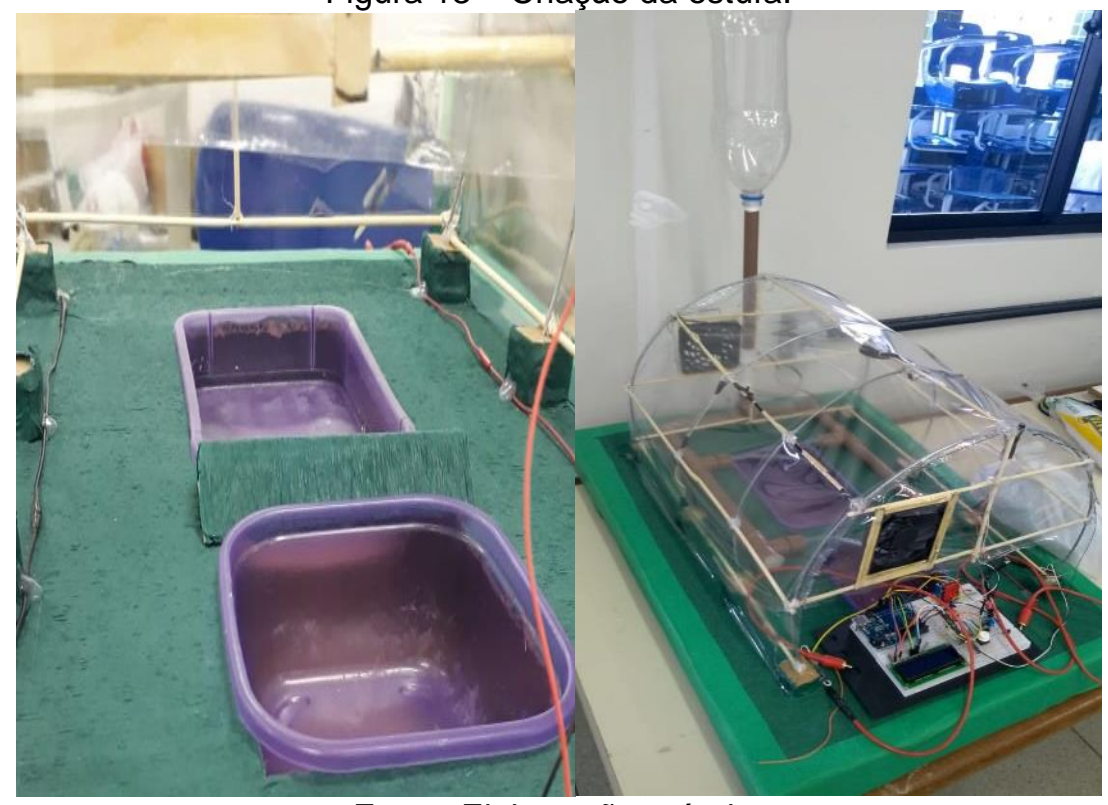

Fonte: Elaboração própria.

Na Figura 14 está apresentada a fonte de alimentação utilizada para alimentar os componentes do sistema de monitoramento e também os equipamentos da estufa (válvula solenoide, coolers e iluminação). Usamos a fonte de alimentação de um DVD quebrado, a carcaça de um roteador, além de: fios, estanho, cola, parafusos e plugs que serviram para construir, dar segurança e conduzir a corrente necessária para alimentação do nosso projeto.

Figura 14 - Fonte de alimentação do projeto.

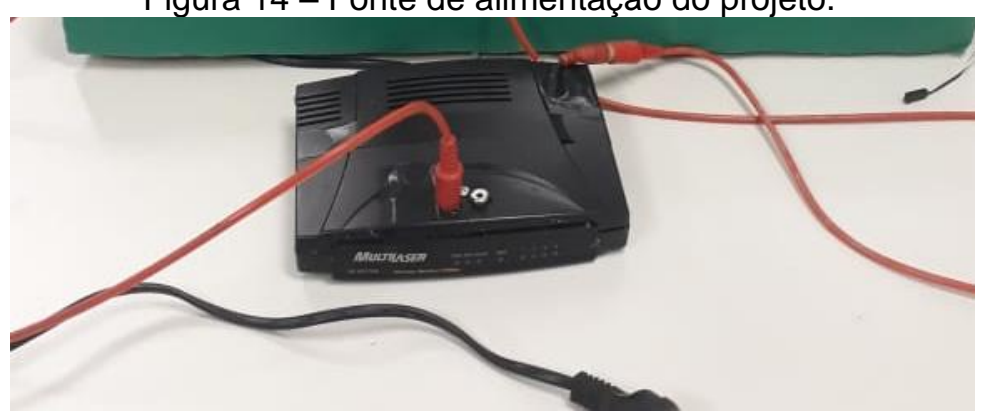

Fonte: Elaboração própria.

Na Figura 15 está apresentado o funcionamento do projeto inserido na estufa com a fonte de alimentação. Utilizou-se o recipiente apresentado na Figura 12 para simular uma plantação. 
Figura 15 - Protótipo com fonte de alimentação.

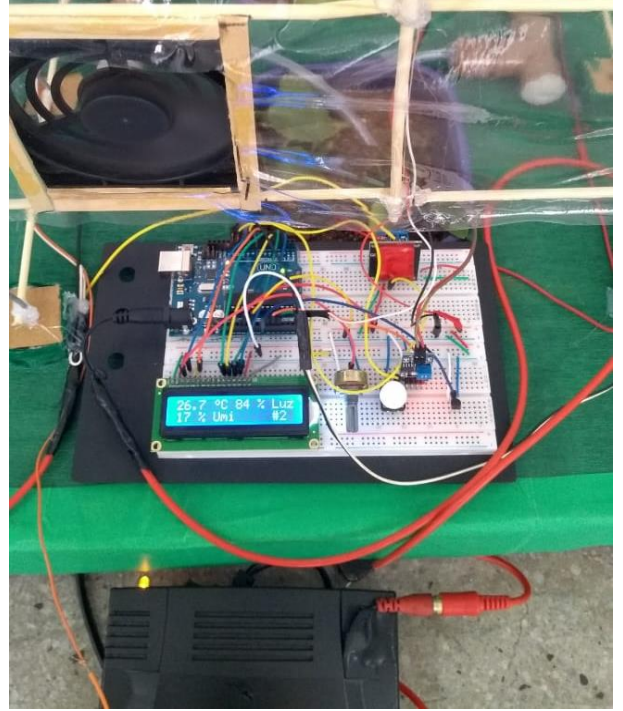

Fonte: Elaboração própria.

\subsection{Continuidade}

Como continuidade do trabalho, pretendemos:

- adicionar uma comunicação com o celular através do módulo bluetooth como observado na Figura 16 e desenvolver um aplicativo para celular de modo que o usuário tenha mais conforto no monitoramento da sua plantação.

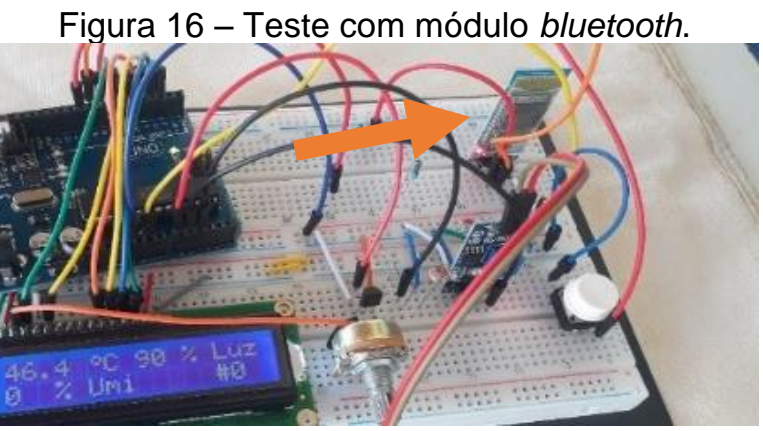

Fonte: Elaboração própria.

- criar uma placa de circuito impresso (PCB) para a soldagem dos sensores, pois desta maneira, será possível evitar erros de leitura provenientes do mal contato dos sensores na protoboard.

- melhorar o código de modo que seja possível: de maneira simples modificar as configurações individuais de cada "modo de cultivo".

- criar um gabinete no qual será utilizado para proteção dos componentes eletrônicos dando ao protótipo um aspecto de produto final.

Na Figura 17, está apresentada a logo que será usada como ícone do aplicativo. 0 mesmo será intitulado Monitor T.U.L que representa a temperatura, umidade e luminosidade. As cores laranja, vermelha e azul marinho representam os dados do ambiente, os quais são coletados pelos sensores, a cor azul claro representa a plataforma Arduino e a cor branca juntamente com os números em binário representam a conversão destes dados. 
Figura 17 - Logo: monitoramento T.U.L

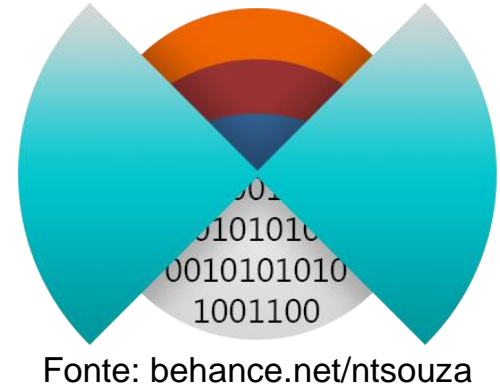

Os alunos envolvidos no projeto relatam que: "Todo o desenvolvimento levou a inúmeros benefícios. Um deles, por exemplo, refere-se aos agricultores de baixa renda. Pois eles terão a possibilidade de monitorar suas plantações afim de tomarem iniciativas que reduzam possíveis danos causados por pestes e até pelo próprio meio ambiente. Outro benefício é a possibilidade de aliar o conhecimento teórico estudado em sala de aula com a prática, obtendo assim uma melhor percepção de como a teoria explica a prática e de como ambas são de extrema importância para carreira de um técnico em eletrotécnica pois, com a prática é possível observar as dificuldades em se desenvolver um projeto. Com esse estudo também foi possível treinar melhor as habilidades de resolução de problemas que haverá na carreira profissional".

\section{CONSIDERAÇÕES FINAIS}

Este trabalho apresentou o desenvolvimento de um protótipo para o monitoramento de temperatura, umidade e luminosidade para uso em pequenas plantações do sertão paraibano. Os resultados obtidos foram satisfatórios, pois foi possível realizar a caracterização dos sensores utilizados para a obtenção dos dados com base no custo, disponibilidade, simplicidade na utilização e facilidade na integração com o Arduino de acordo com as necessidades do agricultor.

O desenvolvimento de atividades práticas aliadas à aprendizagem baseada em problemas - ABP que foi empregada no trabalho apresentado nesse artigo mostrou-se bastante satisfatória. Os alunos aliaram o conhecimento teórico, adquirido em algumas disciplinas, à prática e desenvolveram várias habilidades, como o trabalho em equipe, interação com pessoas da comunidade local, iniciativa e empatia.

Como continuidade da pesquisa busca-se desenvolver o protótipo em uma placa de circuito impresso, além de finalizar o desenvolvimento do aplicativo para celulares pois assim, o usuário, pode receber os dados coletados pelo monitor T.U.L no seu aparelho celular, proporcionando mais conforto e também maior agilidade para tomada de decisões.

\section{Agradecimentos}

Os autores agradecem ao IFPB pelo suporte técnico e financeiro.

\section{REFERÊNCIAS}

\section{Livros:}

Um autor: 
WERNECK, M. M.; Transdutores e Interfaces; Rio de Janeiro; Livros Técnicos e Científicos Editora S. A; 1996.

WENDLING, Marcelo. Sensores. 2010. Disponível em:

http://www2.feg.unesp.br/Home/PaginasPessoais/ProfMarceloWendling/4---sensores-

v2.0.pdf. Acesso em 23 fevereiro 2019.

Dois autores:

OLIVEIRA, Cláudio Luiz Vieira; ZANETTI, Humberto Augusto Piovesana. Arduino Descomplicado: como elaborar projetos de eletrônica. São Paulo: Editora Érica, 2015

\section{Capítulos de Livros:}

STEIDLE NETO, A. J. et al. Avaliação da transmissão de dados de temperatura no

Sistema 1- WIRE ${ }^{\text {TM }}$. Engenharia Agrícola, Jaboticabal, v. 25, n. 1, p. 29-36, jan./abr. 2005.

Monografias, dissertações e teses:

SOUZA Mendes, Paulo Cesar. Caracterização de um sensor para

medição de umidade do solo com termo-resistor a temperatura constante. Universidade Federal da Bahia, Bahia, Brasil, Tese de Doutorado, 2006.

SOUZA, S. C.; DOURADO, L. APRENDIZAGEM BASEADA EM PROBLEMAS (ABP): UM MÉTODO DE APRENDIZAGEM INOVADOR PARA O ENSINO EDUCATIVO.

SOUZA \& DOURADO (2015): Artigo submetido em março/2015 e aceito em setembro/2015.

Artigos de jornais:

SHASHWATHI, N; BORKOTOKY, P; SUHAS K. Smart Farming: A Step towards TechnoSavvy Agriculture. 2012. Disponível em:

http://research.ijcaonline.org/volume57/number18/pxc3883773.pdf. Acesso em 04 Março 2019

\section{Trabalhos em eventos}

EVANGELISTA, T. S. et al. Protótipo de um dinamômetro usando Arduino e material de sucata para aulas de laboratório de física como estudo de caso da ABP.

Anais: XLIV Congresso Brasileiro de Educação em Engenharia (COBENGE 2016), Natal (Brasil), 2016.

\section{Internet:}

Arduino. 0 que é Arduino?, 2019. Disponível em: https://www.arduino.cc/en/Guide/Introduction. Acesso em: 21 fevereiro 2019.

Instituto Brasileiro de Geografia e Estatística (IBGE) Disponível em: https://cidades.ibge.gov.br/brasil/pb/patos/panorama. Acesso em 02 março 2019.

Instituto Nacional de Meteorologia (INMET) Disponível em: http://www.inmet.gov.br/portal/index.php?r=clima/normaisclimatologicas. Acesso em 04 março 2019.

LM35 Precision Centigrade Temperature Sensors datasheet. Disponível em: http://www.ti.com/lit/ds/symlink/lm35.pdf. Acesso em 20 fevereiro 2019. 
Sensor

de

luminosidade,

LDR.

Disponível

em:

https://www.eletronicacastro.com.br/ldr/9617-sensor-ldr-7mm-0000000096171.html.

Acesso em 23 fevereiro 2019.

Sensor De Umidade Do Solo Resistivo. Disponível em:

https://produto.mercadolivre.com.br/MLB-962779536-5-pecas-sensor-de-umidade-do-

solo-resistivo-higrmetro-

JM?matt tool=88344885\&matt word\&gclid=CjwKCAiA2fjiBRAjEiwAuewS V ft2xnfkXxb

XyDOAsClHvwDtPSYZe8AvMalJzAZ 5raEEnl UdlxoCObIQAvD BwE\&quantity=1.

Acesso em 23 fevereiro 2019.

\title{
TEMPERATURE, HUMIDITY AND LUMINOSITY MONITORING SYSTEM FOR USE ON SMALL PLANTATIONS IN THE PARAÍBA'S COUNTRYSIDE
}

\begin{abstract}
The constant technological evolution makes it possible to use electronics to improve working conditions, in order to avoid waste, losses and increase productivity. In the area of agriculture, electronics can be used to obtain the necessary information that a plant needs to develop, such as temperature, soil moisture, light, among others. In this scenario, the main focus of this study was to develop a monitoring system using sensors and Arduino that would enable the low-income user to have the possibility to improve their cultivation environment. Using the Tinkercad platform it was possible to carry out the simulations that monitor the physical phenomena and in the future become a prototype called Monitor T.U.L, this control is done using the Arduino UNO and some sensors. With that, it was possible to reach the goal of a prototype that proved to be very effective in data collection and automation using relays, low cost and easy integration with Arduino. To continue, we seek to improve it, with improved code, food and the creation of an application that will work with the t.u.I monitor, making it even easier to collect and transmit data.
\end{abstract}

Keywords: Arduino. Sensors. Monitoring. Paraíba’s countryside. 